\title{
Application of Machine Learning to Predict Cervical Lymph Node Metastasis from Micropapillary Thyroid Carcinoma with Ultrasound
}

\section{Bo Shen}

The First people's Hospital of Taicang

Chao Zhou

The First People's Hospital of Taicang

Chaoli Xu ( $\square$ sanyecao0104@163.com )

Nanjing Jinling Hospital https://orcid.org/0000-0003-3374-9897

\section{Bin Yang}

Nanjing Jinling Hospital

Xiaoman Wu

The First People's Hospital of Taicang

\section{Xiaodan Fu}

The First People's Hospital of Taicang

\section{Siyue Liu}

The First People's Hospital of Taicang

Jiaying Sun

The First People's Hospital of Taicang

Qirui Zhang

Nanjing Jinling Hospital

Zheng Zhu

The First People's Hospital of Taicang

\section{Research}

Keywords: Thyroid micropapillary carcinoma, Central lymph node metastasis, Ultrasound, Anteroposterior/transverse diameter ratio, Nomogram

Posted Date: November 9th, 2021

DOI: https://doi.org/10.21203/rs.3.rs-1050834/v1

License: (c) (i) This work is licensed under a Creative Commons Attribution 4.0 International License. Read Full License 


\section{Abstract}

Background This study aims to determine the prediction performance of a machine learning-based clinical model for cervical lymph node metastasis (CLNM) in micropapillary thyroid carcinoma (MPTC) with ultrasound (US).

Methods Patients with MPTC who underwent total or hemithyroidectomy with unilateral or bilateral prophylactic central neck dissection were included $(n=692)$. Nodal status was pathologically determined. Clinical and US features and thyroid function markers were extracted to build a random forest model. A nomogram with the significant predictive risk factors from multivariable logistic regression analysis was built to visualize hazard rates. Finally, the predictive performances of the models were compared.

Results Overall, 332 patients (47.98\%) had CLNM. In multiple logistic regression, the strong predictive risk factors for CLNM were younger age, larger anteroposterior diameter, lower anteroposterior/transverse diameter $(\mathrm{A} / \mathrm{T})$ ratio, and higher thyroglobulin $(T G)$ concentration $(P<0.05)$. The random forest and nomogram models showed good predictive performance with the area under the curves (AUCs) of 0.836 and 0.780 , respectively, which were significantly higher than those without A/T ratio in the models (AUCs: 0.807 vs. 0.722 , all $P<0.05$ ). The AUC of the $A / T$ ratio as a single feature for predicting CLNM was 0.744 , while $\mathrm{A} / \mathrm{T}$ ratio $(\leq 0.828)$ combined with anteroposterior diameter $(\geq 10 \mathrm{~mm})$ yielded a higher AUC of 0.754 for predicting CLNM.

Conclusions The machine learning-based clinical model with US had a good predictive performance for CLNM in MPTC patients. This clinical model may facilitate surgical decision-making for MPTC, especially regarding whether cervical lymph node dissection is warranted.

\section{Background}

Micropapillary thyroid cancer (MPTC), which refers to a papillary cancer that is $10 \mathrm{~mm}$ or less in its maximal diameter, is widespread, with an estimated prevalence ranging from $6-35 \%$ of thyroid carcinomas. [1-3] Although studies have shown that MPTC is an indolent tumor, pathologic examinations of routinely dissected neck node specimens have indicated that neck lymph node metastasis, especially central lymph node metastasis (CLNM), can occur in $47.0-64.1 \%$ of patients. [4, 5] Furthermore, the risk of recurrence was estimated to be 3.33-fold higher in patients with CLNM than in those without CLNM. [6, 7] Patients with recurrence generally need reoperation, which significantly increases the risk for temporary parathyroid laryngeal damage and injury to the laryngeal nerves and cervical plexus, causing additional complications. [8] Therefore, to determine the involvement of CLNM in MPTC is not negligible.

Preoperative ultrasonography (US) is the most sensitive method for detecting CLNM and, as such, is recommended as part of the standard preoperative workup by the American Thyroid Association (ATA) and the National Comprehensive Cancer Network (NCCN). [9-11] However, the identification of CLNM by 
US has encountered significant challenges. Preoperative US can only detect $20-31 \%$ of CLNM and may only change the surgical management of $20 \%$ of patients. [12-14] There is an urgent need for a nondestructive and efficient method for predicting the risk of CLNM in MPTC patients to guide the clinical diagnosis and treatment process.

Machine learning $(\mathrm{ML})$ is a novel computer-based method for data analysis that can find more interactions between variables and outcomes by learning from dataset patterns, thus providing improved diagnostic and prognostic accuracy over conventional statistical methods. Studies have shown that ML has a high diagnostic performance in evaluating CLNM in patients with thyroid cancer, with an area under the curve (AUC) of 0.67 to 0.78 . [15-17] However, the prediction performance for CLNM was not ideal since completeness of the extracted image features is difficult to guarantee. Furthermore, few studies have focused on the predictive performance of ML in evaluating CLNM in MPTC patients.

In the current study, we hypothesized that ML models based on US could achieve a satisfactory performance in predicting CLNM in MPTC patients. The purpose of this study was, first, to develop an ML-based model to distinguish CLNM from non-CLNM based on preoperative US images. Second, this study aimed to select the significant risk factors for CLNM using multiple logistic regression analysis. Third, a nomogram was constructed to visualize the predictions.

\section{Materials And Methods}

\section{Participants}

This retrospective study was approved by the ethics committee at Jinling Hospital, and all patients provided written informed consent. A total of 692 patients who had pathological MPTC and underwent lobectomy or total or near-total thyroidectomy and central neck dissection (CLND), with or without lateral neck dissection (LND) from January 2014 to June 2021 were studied. The exclusion criteria were as follows: (1) a previous history of thyroidectomy; (2) no histologically proven MPTC, (2) more than one MPTC lesion, (3) no lymph nodes removed and inadequate preoperative blood test report, (4) pathologically confirmed tumor size $>1 \mathrm{~cm}$, or presence of a skip metastasis, and (5) history or coexistence of other head and neck cancers.

\section{Us Examination}

All of the included patients underwent US scanning before surgery. High-quality US images were acquired with commercial US devices (IU22, Philips Healthcare, Bothell, WA, USA; Logic 9, GE Healthcare, Milwaukee, WI, USA) with linear probes (3-12 MHz, centered at $10 \mathrm{MHz}$ ). Before collecting US data, all US radiologists involved in the acquisition of US images had more than 5 years of experience in thyroid US. They underwent rigorous training to standardize the imaging parameter adjustment method and the US scanning procedure of the thyroid according to the AIUM practice guideline for performing thyroid US. [18] It is routinely required to acquire images of the anteroposterior and transverse sections of the target 
nodules for subsequent analysis. All the data were gathered and reviewed for further analysis by two senior US radiologists blinded to the clinical and pathological results, and only the data that passed the quality control examination were included.

\section{Surgical Methods And Histopathologic Examination With Surgical Specimens}

Hemithyroidectomy was performed when a single tumor was confined to a single lobe. Total thyroidectomy was performed when extrathyroidal extension (ETE), or abnormal lymphadenopathy was detected during the preoperative or intraoperative examination. CLND was defined as a level of VI dissection including pre- and paratracheal nodes, precricoid nodes, perithyroidal nodes, and lymph nodes along recurrent laryngeal nerves. CLND was performed on all pathologically proven conventional PTMC patients. LND was defined as the excision of the lateral neck lymph nodes including modified radical neck dissection and selective neck dissection. Therapeutic LND was performed in cases with biopsyproven or ultrasound-suspicious lateral cervical lymphadenopathy. Surgical specimens were microscopically examined by two or more experienced pathologists. Histopathologic examination included the cell type of the lesion, the primary tumor size (measured as the longest diameter of the largest lesion), ETE, lymphovascular invasion, intrathyroidal spreading, regional lymph node metastasis, and underlying conditions of the thyroid such as Hashimoto thyroiditis and nodular goiter. Intrathyroidal spreading referred to a major thyroid carcinoma with surrounding scattered small lesions, with features of heterotypic cells, psammoma bodies, and lymphatic vessel invasion.

\section{Feature Extraction}

The variables used for model development included both clinical and image features. The clinical variables included sex, age, and serum calcitonin (CT), parathyroid hormone (PTH), thyroglobulin (TG), thyroid-stimulating hormone (TSH), triiodothyronine (T3), free triiodothyronine (FT3), thyroxine (T4), and free thyroxine (FT4) levels; the imaging variables included thyroid size and echogenicity, nodule size, anteroposterior/transverse diameter $(\mathrm{A} / \mathrm{T})$ ratio, nodule position, location within the lobe, nodule morphology, nodule boundary, nodule margin, nodule echogenicity, posterior echo attenuation, side shadowing, halo sign, lesion calcification and blood flow, and surrounding thyroid tissue type (normal, Hashimoto thyroiditis, or nodular goiter). These features were extracted and used to estimate the probability of CLNM (detailed in Table 1). Two radiologists read the images and performed feature extraction. If discrepancies occurred, an agreement was reached through discussion. The missing data rates of all features were less than $10 \%$. Regarding missing data, mean interpolation was used for continuous variables, and mode interpolation was used for rank or categorical variables. The categorical variables were then coded with features, and 53 features were obtained. 
Table 1

Definitions of the clinical and US features

\section{Features Value Definition}

Sex

$\begin{array}{lll}\text { Female } & 1 & - \\ \text { Male } & 2 & -\end{array}$

Age $\quad-\quad$ Age when the lesion was pathologically confirmed for the first time

Thyroid size

Transverse $\quad-\quad$ The size of the thyroid gland in the transverse section

diameter

(right/left)

Anteroposterior - $\quad$ The size of the thyroid gland in the anteroposterior section

diameter

(right/left)

Isthmus

- $\quad$ The size of the thyroid isthmus in the longitudinal section

\section{Background \\ parenchymal \\ echogenicity}

Normal parenchymal

echogenicity

Abnormal parenchymal

echogenicity
$1 \quad$ Homogenous echogenicity and relative hyperechogenicity compared with the adjacent sternohyoid, sternothyroid, omohyoid, and sternocleidomastoid muscles

2 Irregular echotexture, micronodularity, and diffuse or focal

hypoechogenic lesions and nodules

Tumor position

$\begin{array}{lll}\text { Right lobe } & 1 & - \\ \text { Left lobe } & 2 & - \\ \text { Isthmus } & 3 & -\end{array}$

Location within

the lobe

\begin{tabular}{|c|c|c|}
\hline Upper lobe & 1 & The nodule was in the upper $1 / 3$ of the lobe \\
\hline Mid lobe & 2 & The nodule was in the middle of the lobe \\
\hline Lower lobe & 3 & The nodule was in the lower $1 / 3$ of the lobe \\
\hline Isthmus & 4 & The nodule was in the isthmus of the thyroid \\
\hline
\end{tabular}

Tumor size 


\begin{tabular}{|c|c|c|}
\hline Features & Value & Definition \\
\hline $\begin{array}{l}\text { Transverse } \\
\text { diameter }\end{array}$ & - & The size of the nodule in the transverse section \\
\hline $\begin{array}{l}\text { Anteroposterior } \\
\text { diameter }\end{array}$ & - & The size of the nodule in the anteroposterior section \\
\hline $\mathrm{A} / \mathrm{T}$ ratio & - & $\begin{array}{l}\text { The ratio of the anteroposterior and transverse diameters of the } \\
\text { nodules }\end{array}$ \\
\hline \multicolumn{3}{|l|}{$\begin{array}{l}\text { Tumor } \\
\text { morphology }\end{array}$} \\
\hline Regular & 1 & $\begin{array}{l}\text { An oval (egg-shaped or elliptical) or round (spherical, ball-shaped) } \\
\text { mass }\end{array}$ \\
\hline Irregular & 2 & Microlobulated shape \\
\hline \multicolumn{3}{|l|}{ Tumor boundary } \\
\hline Clear & 1 & The demarcation was clear \\
\hline Unclear & 2 & $\begin{array}{l}\text { The demarcation was unclear without an abrupt transition between } \\
\text { the lesion and the surrounding tissue }\end{array}$ \\
\hline \multicolumn{3}{|l|}{ Tumor margin } \\
\hline Clear & 1 & $\begin{array}{l}\text { The margin was well defined and clear with an abrupt transition } \\
\text { between the lesion and the surrounding tissue }\end{array}$ \\
\hline Unclear & 2 & $\begin{array}{l}\text { The margin was characterized as indistinct, angular, microlobulated, } \\
\text { or spiculated }\end{array}$ \\
\hline \multicolumn{3}{|l|}{ Echogenicity } \\
\hline $\begin{array}{l}\text { Markedly } \\
\text { hypoechoic }\end{array}$ & 1 & The mass has significantly decreased echogenicity compared to fat \\
\hline Hypoechoic & 2 & The mass has decreased echogenicity compared with fat \\
\hline $\begin{array}{l}\text { Isoechoic/mixed } \\
\text { echoic }\end{array}$ & 3 & $\begin{array}{l}\text { The mass has the same or slightly increased echogenicity compared } \\
\text { with fat/a complex mass containing both anechoic (cystic) and } \\
\text { echogenic (solid) components }\end{array}$ \\
\hline \multicolumn{3}{|l|}{$\begin{array}{l}\text { Posterior echo } \\
\text { attenuation }\end{array}$} \\
\hline No & 1 & $\begin{array}{l}\text { No shadowing was present deep in the mass. The echogenicity of the } \\
\text { area immediately behind the mass was not different from that of the } \\
\text { adjacent tissue at the same depth }\end{array}$ \\
\hline Yes & 2 & $\begin{array}{l}\text { Shadowing, i.e., posterior attenuation of acoustic transmission. } \\
\text { Sonographically, the area posterior to the mass appeared darker }\end{array}$ \\
\hline \multicolumn{3}{|l|}{ Side shadowing } \\
\hline No & 1 & Without side shadowing \\
\hline
\end{tabular}




\begin{tabular}{|c|c|c|}
\hline Features & Value & Definition \\
\hline Yes & 2 & $\begin{array}{l}\text { Posterior attenuation of the acoustic transmission from both sides of } \\
\text { the lesion }\end{array}$ \\
\hline \multicolumn{3}{|l|}{ Halo sign } \\
\hline No & 1 & No band bridged by an echogenic transition zone could be observed \\
\hline Yes & 2 & A band bridged by an echogenic transition zone could be observed \\
\hline \multicolumn{3}{|l|}{ Calcifications } \\
\hline None & 1 & No calcifications \\
\hline Microcalcifications & 2 & Microcalcifications embedded in the mass area were well depicted \\
\hline $\begin{array}{l}\text { Coarse } \\
\text { calcifications }\end{array}$ & 3 & $\begin{array}{l}\text { Macrocalcifications, defined as coarse calcifications } 0.5 \mathrm{~mm} \text { or } \\
\text { greater in size, were depicted }\end{array}$ \\
\hline \multicolumn{3}{|l|}{$\begin{array}{l}\text { Color Doppler flow } \\
\text { imaging (CDFI) } \\
\text { grade }\end{array}$} \\
\hline Adler 0 & 0 & No vascularity \\
\hline Adler 1 & 1 & Little vascularity \\
\hline Adler 2 & 2 & Vascularity present immediately adjacent to the lesion \\
\hline Adler 3 & 3 & Diffusely increased vascularity surrounding the lesion \\
\hline \multicolumn{3}{|l|}{$\begin{array}{l}\text { Surrounding } \\
\text { thyroid tissues }\end{array}$} \\
\hline Normal & 1 & Pathologically confirmed as normal thyroid tissue \\
\hline Nodular goiter & 2 & Pathologically confirmed as normal nodular goiter \\
\hline $\begin{array}{l}\text { Hashimoto } \\
\text { thyroiditis }\end{array}$ & 3 & Pathologically confirmed as Hashimoto thyroiditis \\
\hline
\end{tabular}

SPSS 22.0 and R (http://www.R-project.org) software were used for statistical analysis. Univariate analysis was performed, and variables with statistical significance were further included in the multivariate logistic models. Multivariate analysis was performed with binary logistic regression analysis to identify independent risk factors for CLNM. Statistical significance was considered when $P<0.05$.

\section{Model Construction}

First, the random forest algorithm was used to build the classifier model and evaluate all of the features for their ability to predict CLNM. Then, the weighted features were screened out according to their respective coefficients. The feature selection process used the least absolute shrinkage and selection 
operator algorithm with a penalty term called L1-norm (C-index was set as 1.00). Finally, a model was constructed using 5 -fold cross-validation and was independently tested. Calibration curves were plotted to assess the calibration of the random forest models, accompanied by the Hosmer-Lemeshow test. (A significant result implied that the model does not calibrate perfectly.) Decision curve analysis was conducted to determine the clinical usefulness of the model by quantifying the net benefits at different threshold probabilities.

Second, to provide clinicians with a quantitative tool to predict an individual's probability of CLNM, we built a nomogram based on the risk factors obtained by multivariate logistic regression analyses. To choose the most significant parameters for predicting CLNM, we chose the top 4 parameters associated with the highest risk, which were also significant risk factors from multivariate logistic regression. The nomogram was plotted using R with the "Hmisc" package.

Finally, we specifically evaluated the diagnostic performance of the anteroposterior diameter, A/T ratio, and combination of anteroposterior diameter and $A / T$ ratio on US images as single features and determined a cutoff point for tumor size with a high specificity of $95 \%$.

The diagnostic performance of each model was evaluated by using receiver operating characteristic (ROC) curves and their corresponding AUCs. The differences between AUCs were compared using Delong analysis. The optimal cutoff value, accuracy, sensitivity, specificity, positive predictive value (PPV), and negative predictive value (NPV) were calculated to assess the predictive ability of each model.

\section{Results}

\section{Univariate analysis}

Of the 692 patients, 332 (47.98\%) had MPTC with CLNM (Table 2). Patients with CLNM were significantly younger than those without CLNM (39.43 \pm 11.56 years vs. $44.89 \pm 11.14$ years, $P<0.05)$. There was a statistically significant difference in TG level between the patients with and without CLNM. However, there were no significant differences in sex or CT, PTH, T3, FT3, FT4, TSH, and T4 values between the two groups $(P>0.05)$.

The tumors of patients with CLNM had a larger anteroposterior diameter $(9.43 \pm 4.44 \mathrm{~mm}$ vs. $6.89 \pm 2.67$ $\mathrm{mm}, P<0.05)$, larger transverse diameter $(7.22 \pm 2.72 \mathrm{~mm}$ vs. $6.81 \pm 2.44 \mathrm{~mm}, P<0.05)$, and lower $\mathrm{A} / \mathrm{T}$ ratio than those of patients without CLNM $(0.81 \pm 0.20$ vs. $1.03 \pm 0.26, P<0.05)$. Furthermore, the nodules of patients with CLNM were more likely to have irregular morphology, unclear boundaries, unclear margins, an isoechoic/mixed echoic appearance, posterior echo attenuation, and coexisting calcifications and blood flow signals $(P<0.05)$. The other characteristics of the thyroid nodules, including coexisting side shadowing and halo signs, were not different between groups $(P>0.05)$. There were no differences in thyroid size, echogenicity, or concomitant diseases between groups $(P>0.05)$. 
Table 2

Univariate regression analysis of the clinical and US characteristics of the patients with thyroid cancer

\begin{tabular}{|c|c|c|c|c|c|}
\hline Variables & $\begin{array}{l}\text { All patients } \\
(n=692)\end{array}$ & $\begin{array}{l}\text { Without CLNM } \\
(n=360)\end{array}$ & $\begin{array}{l}\text { With CLNM } \\
(n=332)\end{array}$ & $x^{2} / t$ & $\begin{array}{l}P \\
\text { Value }\end{array}$ \\
\hline Age (year) & $42.27 \pm 11.66$ & $44.89 \pm 11.14$ & $39.43 \pm 11.56$ & 6.330 & 0.000 \\
\hline Sex & & & & 1.028 & 0.311 \\
\hline Male & $239(34.54 \%)$ & $118(17.05 \%)$ & $121(17.49 \%)$ & & \\
\hline Female & $453(65.46 \%)$ & $242(34.97 \%)$ & $211(30.49 \%)$ & & \\
\hline Thyroid echogenicity & & & & 1.858 & 0.173 \\
\hline $\begin{array}{l}\text { Normal parenchymal } \\
\text { echogenicity }\end{array}$ & $609(88.01 \%)$ & $311(44.94 \%)$ & $298(43.07 \%)$ & & \\
\hline $\begin{array}{l}\text { Abnormal parenchymal } \\
\text { echogenicity }\end{array}$ & $83(11.99 \%)$ & $49(7.08 \%)$ & $34(4.91 \%)$ & & \\
\hline \multicolumn{6}{|l|}{ Thyroid size (mm) } \\
\hline Isthmus & $2.68 \pm 0.84$ & $2.66 \pm 0.90$ & $2.70 \pm 0.78$ & -0.678 & 0.498 \\
\hline $\begin{array}{l}\text { Transverse diameter } \\
\text { (right) }\end{array}$ & $17.75 \pm 4.86$ & $18.03 \pm 6.15$ & $17.45 \pm 2.85$ & 1.556 & 0.120 \\
\hline $\begin{array}{l}\text { Anteroposterior diameter } \\
\text { (right) }\end{array}$ & $14.73 \pm 3.00$ & $14.75 \pm 3.15$ & $14.71 \pm 2.84$ & 0.186 & 0.852 \\
\hline Transverse diameter (left) & $17.15 \pm 2.90$ & $17.29 \pm 2.97$ & $16.99 \pm 2.81$ & 1.345 & 0.179 \\
\hline $\begin{array}{l}\text { Anteroposterior diameter } \\
\text { (left) }\end{array}$ & $14.12 \pm 2.94$ & $14.09 \pm 2.89$ & $14.16 \pm 2.99$ & -0.326 & 0.745 \\
\hline Nodule position & & & & 1.771 & 0.425 \\
\hline Right lobe & $364(52.60 \%)$ & $181(26.16 \%)$ & $183(26.45 \%)$ & & \\
\hline Left lobe & $318(45.95 \%)$ & $174(25.14 \%)$ & $144(20.81 \%)$ & & \\
\hline Isthmus & $10(1.45 \%)$ & $5(0.725 \%)$ & $5(0.725 \%)$ & & \\
\hline Location within lobe & & & & 2.316 & 0.509 \\
\hline Upper lobe & $147(21.24 \%)$ & $81(11.70 \%)$ & $66(9.54 \%)$ & & \\
\hline Mid lobe & $312(45.09 \%)$ & $167(24.13 \%)$ & $145(20.96 \%)$ & & \\
\hline Lower lobe & $223(32.22 \%)$ & 107 (15.46\%) & $116(16.76 \%)$ & & \\
\hline Isthmus & $10(1.45 \%)$ & $5(0.725 \%)$ & $5(0.725 \%)$ & & \\
\hline
\end{tabular}




\begin{tabular}{|c|c|c|c|c|c|}
\hline Variables & $\begin{array}{l}\text { All patients } \\
(\mathrm{n}=692)\end{array}$ & $\begin{array}{l}\text { Without CLNM } \\
(n=360)\end{array}$ & $\begin{array}{l}\text { With CLNM } \\
(n=332)\end{array}$ & $X^{2} / t$ & $\begin{array}{l}P \\
\text { Value }\end{array}$ \\
\hline $\begin{array}{l}\text { Anteroposterior diameter } \\
(\mathrm{mm})\end{array}$ & $8.11 \pm 3.84$ & $6.89 \pm 2.67$ & $9.43 \pm 4.44$ & -9.229 & 0.000 \\
\hline $\begin{array}{l}\text { Transverse diameter } \\
(\mathrm{mm})\end{array}$ & $7.01 \pm 2.58$ & $6.81 \pm 2.44$ & $7.22 \pm 2.72$ & -2.102 & 0.036 \\
\hline $\mathrm{A} / \mathrm{T}$ ratio & $0.92 \pm 0.26$ & $1.03 \pm 0.26$ & $0.81 \pm 0.20$ & 12.006 & 0.000 \\
\hline Morphology & & & & 7.137 & 0.008 \\
\hline Regular & $126(18.21 \%)$ & $52(7.51 \%)$ & $74(10.70 \%)$ & & \\
\hline Irregular & $566(81.79 \%)$ & $308(44.51 \%)$ & $258(37.28 \%)$ & & \\
\hline Boundary & & & & 3.902 & 0.048 \\
\hline Clear & $129(18.64 \%)$ & $57(8.24 \%)$ & $72(10.40 \%)$ & & \\
\hline Unclear & $563(81.36 \%)$ & $303(43.79 \%)$ & $260(37.57 \%)$ & & \\
\hline Margin & & & & 4.936 & 0.026 \\
\hline Clear & $90(13.01 \%)$ & $37(5.35 \%)$ & $53(7.66 \%)$ & & \\
\hline Unclear & $602(86.99 \%)$ & $323(46.67 \%)$ & $279(40.32 \%)$ & & \\
\hline Echogenicity & & & & 13.419 & 0.001 \\
\hline Markedly hypoechoic & $51(7.37 \%)$ & $34(4.91 \%)$ & $17(2.46 \%)$ & & \\
\hline Hypoechoic & $612(88.44 \%)$ & $319(46.10 \%)$ & $293(42.34 \%)$ & & \\
\hline Isoechoic/mixed echoic & $29(4.19 \%)$ & $7(1.01 \%)$ & $22(3.18 \%)$ & & \\
\hline $\begin{array}{l}\text { Posterior echo } \\
\text { attenuation }\end{array}$ & & & & 8.267 & 0.004 \\
\hline No & $568(82.08 \%)$ & $281(40.61 \%)$ & $287(41.47 \%)$ & & \\
\hline Yes & $124(17.92 \%)$ & $79(11.42 \%)$ & $45(6.50 \%)$ & & \\
\hline Shadowing & & & & 0.812 & 0.367 \\
\hline No & $648(93.64 \%)$ & $340(49.13 \%)$ & $308(44.51 \%)$ & & \\
\hline Yes & $44(6.36 \%)$ & $20(2.89 \%)$ & $24(3.47 \%)$ & & \\
\hline Halo sign & & & & 0.019 & 0.892 \\
\hline No & 645 (93.21\%) & $336(48.55 \%)$ & 309 (44.66\%) & & \\
\hline Yes & $47(6.79 \%)$ & $24(3.47 \%)$ & $23(3.32 \%)$ & & \\
\hline Calcifications & & & & & \\
\hline
\end{tabular}




\begin{tabular}{|llllll|}
\hline Variables & $\begin{array}{l}\text { All patients } \\
(\mathbf{n = 6 9 2})\end{array}$ & $\begin{array}{l}\text { Without CLNM } \\
(\mathbf{n = 3 6 0 )}\end{array}$ & $\begin{array}{l}\text { With CLNM } \\
(\mathbf{n = 3 3 2})\end{array}$ & $X^{2} / t$ & $\begin{array}{c}\boldsymbol{P} \\
\text { Value }\end{array}$ \\
\hline None & $323(46.68 \%)$ & $186(26.88 \%)$ & $137(19.80 \%)$ & 8.085 & 0.018 \\
\hline Microcalcifications & $249(35.98 \%)$ & $114(16.47 \%)$ & $135(19.51 \%)$ & & \\
\hline Coarse calcifications & $120(17.34 \%)$ & $60(8.67 \%)$ & $60(8.67 \%)$ & & \\
\hline CDFI grade & & & & 9.328 & 0.025 \\
\hline Adler 0 & $196(28.32 \%)$ & $117(16.91 \%)$ & $79(11.41 \%)$ & & \\
\hline Adler 1 & $346(50.00 \%)$ & $169(24.42 \%)$ & $177(25.58 \%)$ & & \\
\hline Adler 2 & $89(12.86 \%)$ & $49(7.08 \%)$ & $40(5.78 \%)$ & & \\
\hline Adler 3 & $61(8.82 \%)$ & $25(3.61 \%)$ & $36(5.21 \%)$ & & \\
\hline Surrounding thyroid & & & & & \\
\hline tissues & & & 18.418 & 0.181 \\
\hline Normal & $389(56.20 \%)$ & $206(29.77 \%)$ & $183(26.43 \%)$ & & \\
\hline Nodular goiter & $137(19.80 \%)$ & $62(8.96 \%)$ & $75(10.84 \%)$ & & \\
\hline Hashimoto thyroiditis & $166(24.00 \%)$ & $92(13.29 \%)$ & $74(10.71 \%)$ & & \\
\hline CT (ng/L) & $4.70 \pm 2.33$ & $4.54 \pm 2.33$ & $4.86 \pm 2.31$ & -1.834 & 0.067 \\
\hline PTH (pmol/L) & $4.71 \pm 2.35$ & $4.83 \pm 2.70$ & $4.57 \pm 1.90$ & 1.477 & 0.140 \\
\hline TG ( $\mu$ g/mL) & $18.68 \pm 38.46$ & $16.92 \pm 36.56$ & $20.59 \pm 40.44$ & -3.057 & 0.002 \\
\hline T4 (nmol/L) & $104.98 \pm 19.91$ & $104.32 \pm 18.85$ & $105.71 \pm 21.02$ & -0.918 & 0.359 \\
\hline T3 (nmol/L) & $1.36 \pm 0.30$ & $1.33 \pm 0.26$ & $1.40 \pm 0.34$ & -1.253 & 0.211 \\
\hline TSH (mIU/L) & $2.19 \pm 2.23$ & $2.04 \pm 1.22$ & $2.37 \pm 2.95$ & -1.941 & 0.053 \\
\hline FT3 (pmol/L) & $4.64 \pm 0.61$ & $4.61 \pm 0.59$ & $4.67 \pm 0.64$ & -1.223 & 0.222 \\
\hline FT4 (pmol/L) & $11.04 \pm 1.84$ & $11.09 \pm 1.80$ & $11.00 \pm 1.90$ & 0.667 & 0.505 \\
\hline
\end{tabular}

\section{Multivariate Analysis}

Multiple logistic regression analysis showed that the A/T ratio, anteroposterior diameter, age, and TG concentration were significantly associated with an increased risk for CLNM (Table 3). A lower A/T ratio had a significant positive relationship with the risk of CLNM (OR 1.564, 95\% Cl 1.210-2.020, $P=0.001$ ). A larger anteroposterior diameter had a significant positive relationship with the risk for CLNM (OR 0.702, $95 \% \mathrm{Cl} 0.525-0.939, P=0.017)$. Younger age (OR $0.966,95 \% \mathrm{Cl} 0.951-0.981, P=0.000)$ was 
independently associated with an increased risk for CLNM. Additionally, a higher TG concentration was also associated with a higher risk for CLNM (OR 0.685, 95\% Cl 0.430-1.846, $P=0.019$ ). 
Table 3

Multivariable logistic regression analysis of the clinical and US characteristics of the patients with thyroid cancer

\begin{tabular}{|c|c|c|c|c|c|c|}
\hline Variables & B & $\begin{array}{l}\text { Standard } \\
\text { error }\end{array}$ & Wald & $P$ & OR & $95 \% \mathrm{Cl}$ \\
\hline Age & -0.035 & 0.008 & 18.638 & 0.000 & 0.966 & $\begin{array}{l}0.951- \\
0.981\end{array}$ \\
\hline Anteroposterior diameter (mm) & 0.354 & 0.148 & 5.681 & 0.017 & 0.702 & $\begin{array}{l}0.525- \\
0.939\end{array}$ \\
\hline Transverse diameter (mm) & -0.537 & 1.077 & 0.249 & 0.618 & 0.585 & $\begin{array}{l}0.071- \\
4.825\end{array}$ \\
\hline $\mathrm{A} / \mathrm{T}$ ratio & -0.447 & 0.131 & 11.702 & 0.001 & 1.564 & $\begin{array}{l}1.210- \\
2.020\end{array}$ \\
\hline Morphology (irregular) & 0.159 & 0.299 & 0.283 & 0.595 & 1.172 & $\begin{array}{l}0.653- \\
2.105\end{array}$ \\
\hline Boundary (unclear) & 0.204 & 0.297 & 0.471 & 0.492 & 1.226 & $\begin{array}{l}0.685- \\
2.195\end{array}$ \\
\hline Margin (unclear) & -0.391 & 0.396 & 0.971 & 0.324 & 0.677 & $\begin{array}{l}0.311- \\
1.471\end{array}$ \\
\hline Echogenicity & & & 1.766 & 0.414 & & - \\
\hline Hypoechoic & -0.845 & 0.640 & 1.743 & 0.187 & 0.430 & $\begin{array}{l}0.123- \\
1.506\end{array}$ \\
\hline Isoechoic/mixed echoic & -0.616 & 0.533 & 1.337 & 0.248 & 0.540 & $\begin{array}{l}0.190- \\
1.535\end{array}$ \\
\hline $\begin{array}{l}\text { Posterior echo attenuation } \\
\text { (yes) }\end{array}$ & 0.281 & 0.261 & 1.153 & 0.283 & 1.324 & $\begin{array}{l}0.793- \\
2.210\end{array}$ \\
\hline Calcifications & & & 1.619 & 0.445 & & - \\
\hline Microcalcifications & 0.244 & 0.270 & 0.819 & 0.366 & 1.277 & $\begin{array}{l}0.752- \\
2.166\end{array}$ \\
\hline Coarse calcifications & 0.342 & 0.269 & 1.617 & 0.203 & 1.408 & $\begin{array}{l}0.831- \\
2.384\end{array}$ \\
\hline CDFI grade & & & 3.161 & 0.367 & & - \\
\hline Adler 1 & 0.095 & 0.373 & 0.065 & 0.799 & 1.100 & $\begin{array}{l}0.529- \\
2.284\end{array}$ \\
\hline Adler 2 & 0.392 & 0.351 & 1.249 & 0.264 & 1.480 & $\begin{array}{l}0.744- \\
2.944\end{array}$ \\
\hline Adler 3 & 0.067 & 0.409 & 0.027 & 0.870 & 1.069 & $\begin{array}{l}0.480- \\
2.382\end{array}$ \\
\hline
\end{tabular}




\begin{tabular}{|lllllll|}
\hline Variables & B & $\begin{array}{l}\text { Standard } \\
\text { error }\end{array}$ & Wald & $P$ & OR & $95 \%$ Cl \\
\hline TG $(\mu \mathrm{g} / \mathrm{mL})$ & 0.735 & 0.312 & 5.529 & 0.019 & 0.685 & $\begin{array}{l}0.430- \\
1.846\end{array}$ \\
\hline
\end{tabular}

\section{Diagnostic Performance Of The Random Forest Model}

A total of 53 US and clinical features of the 692 lesions were used to build the random forest model. The top four weighted features, including the $\mathrm{A} / \mathrm{T}$ ratio, anteroposterior diameter, age, and TG, were obtained from feature selection (Figure 1a-e). After 5-fold cross-validation, the model had an AUC of 0.836, which was significantly higher than that of the AUC without A/T ratio $(0.807)(P<0.000)$ (Table 4) (Figure $1 f)$. The calibration curve demonstrated good agreement between the predictions and actual presence of CLNM. The Hosmer-Lemeshow test showed no departure from a perfect fit. The decision curves showed that it would be beneficial to use the model to predict CLNM. 
Table 4

The diagnostic performances of the random forest model, nomogram and tumor size measurements in the dataset

\begin{tabular}{|c|c|c|c|c|c|c|c|}
\hline & $\begin{array}{l}\text { Cutoff } \\
\text { point }\end{array}$ & Accuracy & Sensitivity & Specificity & PPV & NPV & $\begin{array}{l}\text { AUC } \\
{[95 \% \mathrm{Cl}]}\end{array}$ \\
\hline \multirow[t]{2}{*}{$\begin{array}{l}\text { Random forest } \\
\text { model }\end{array}$} & $\begin{array}{l}\text { With } \\
\text { A/T } \\
\text { ratio }\end{array}$ & $74.71 \%$ & $81.11 \%$ & $67.77 \%$ & $73.18 \%$ & $76.79 \%$ & $\begin{array}{l}0.836 \\
(0.807- \\
0.866)^{\star \star}\end{array}$ \\
\hline & $\begin{array}{l}\text { Without } \\
\text { A/T } \\
\text { ratio }\end{array}$ & $73.41 \%$ & $76.39 \%$ & $70.18 \%$ & $73.53 \%$ & $73.27 \%$ & $\begin{array}{l}0.807 \\
(0.775- \\
0.839)^{\star \star}\end{array}$ \\
\hline \multirow[t]{2}{*}{ Nomogram } & $\begin{array}{l}\text { With } \\
\text { A/T } \\
\text { ratio }\end{array}$ & $69.22 \%$ & $72.29 \%$ & $66.40 \%$ & $66.48 \%$ & $72.21 \%$ & $\begin{array}{l}0.780 \\
(0.746- \\
0.814)^{\star \star}\end{array}$ \\
\hline & $\begin{array}{l}\text { Without } \\
\mathrm{A} / \mathrm{T} \\
\text { ratio }\end{array}$ & $67.05 \%$ & $63.55 \%$ & $70.28 \%$ & $66.35 \%$ & $67.65 \%$ & $\begin{array}{l}0.722 \\
(0.687- \\
0.755)^{\star \star}\end{array}$ \\
\hline $\begin{array}{l}\text { Anteroposterior } \\
\text { diameter }\end{array}$ & $10 \mathrm{~mm}$ & $66.18 \%$ & $37.57 \%$ & $95.00 \%$ & $87.84 \%$ & $60.29 \%$ & $\begin{array}{l}0.687 \\
(0.648- \\
0.727)\end{array}$ \\
\hline $\mathrm{A} / \mathrm{T}$ ratio & 0.828 & $68.35 \%$ & $41.80 \%$ & $95.09 \%$ & $90.53 \%$ & $59.27 \%$ & $\begin{array}{l}0.744 \\
(0.708- \\
0.780)\end{array}$ \\
\hline $\begin{array}{l}\text { Anteroposterior } \\
\text { diameter } \\
\text { combined with } \\
\text { A/T ratio }\end{array}$ & $\begin{array}{l}10 \mathrm{~mm}, \\
0.828\end{array}$ & $73.62 \%$ & $48.90 \%$ & $95.04 \%$ & $89.52 \%$ & $68.22 \%$ & $\begin{array}{l}0.754 \\
(0.719- \\
0.790)\end{array}$ \\
\hline
\end{tabular}

\section{Diagnostic Performance Of The Nomogram}

Multiple logistic regression analysis identified four factors, including age, A/T ratio, anteroposterior diameter, and TG, that were highly weighted in the random forest model. These features were applied to develop the nomogram. The nomogram showed a high predictive performance for CLNM with an AUC of 0.780 (95\% Cl: $0.746-0.814$ ), with a sensitivity and specificity of $72.29 \%$ and $66.40 \%$, respectively (Table 4) (Figure 2). However, the nomogram without A/T ratio showed an AUC of 0.722 , with a sensitivity and specificity of $63.55 \%$ and $70.28 \%$, respectively, which was significantly lower than that including $A / T$ ratio $(P=0.001)$.

Diagnostic performance of the anteroposterior diameter, AVT ratio, and combination of anteroposterior diameter and A/T ratio 
Since the tumor size measured by US played an important role in our study, we plotted the ROC curves of the anteroposterior diameter, $\mathrm{A} / \mathrm{T}$ ratio, and combination of anteroposterior diameter and $\mathrm{A} / \mathrm{T}$ ratio to assess their predictive performances. The anteroposterior diameter as a single feature showed an AUC of 0.687 , with the optimal cutoff point of $10 \mathrm{~mm}$, a sensitivity and specificity of $37.57 \%$ and $95.00 \%$, respectively (Table 4) (Figure 3). The A/T ratio as a single feature showed an AUC of 0.745 , which was significantly higher than that of anteroposterior diameter alone $(P=0.0064)$ (Table 4$)$ (Figure 3$)$. With the cutoff point of 0.828 , the $A / T$ ratio yield a sensitivity of $41.80 \%$ and high specificity of $95.09 \%$, respectively. The $A / T$ ratio combined with anteroposterior diameter yielded a significant higher AUC $(0.754,95 \% \mathrm{Cl}: 0.719-0.790)$ than that of anteroposterior diameter alone $(0.687, P<0.001)$. However, there was no significant difference between $A / T$ ratio alone and the combination of $A / T$ ratio and anteroposterior diameter (AUC 0.744 vs. $0.754, P=0.125$ ) (Table 4) (Figure 3).

\section{Discussion}

In the present study, we developed a clinical model and a nomogram based on clinical and US features to estimate the risk of CLNM to improve the surgical management of patients with MPTC. We found that the clinical model had an AUC of 0.836 , and the nomogram showed an AUC of 0.780 for predicting CLNM. Younger age, higher serum TG concentration at diagnosis, larger anteroposterior diameter and lower A/T ratio on US images were associated with CLNM. Notably, the A/T ratio contributes the most important role in CLNM. With an A/T ratio $\leq 0.828$, the predictive performances in clinical model and nomogram were significantly improved along with a high specificity of $95 \%$. These results indicate that this A/T ratio based clinical model may facilitate surgical decision-making for MPTC, especially regarding whether cervical lymph node dissection is warranted.

The management of CLNM in MPTC patients continues to be debated because of the consequences of overtreatment. Therefore, assessing the risk factors for CLNM is essential for MPTC patients. First, younger age is closely related to an increased risk of CLNM. $[6,19,20]$ Furthermore, this increased risk has affects the staging of thyroid cancer. According to the American Joint Committee on Cancer (AJCC) (8th edition), an increase in the age cutoff point from 45 years (7th edition) to 55 years leads to the downstaging of a significant number (23.8\%) of patients, [21] which fully explains the importance of age in the clinical staging of MPTC and suggests that CLNM should be considered in younger patients. Our study indicated that younger age was related to the increased risk of CLNM in conventional MPTC, which was consistent with previous reported. $[6,19,20]$ Moreover, in the current study age predicted a high volume of positive CLNM, thus supporting the routine CLND being encouraged for younger MPTC patients. However, larger sample size studies still needed to be investigated.

Tumor size, specifically a largest diameter $\otimes 5 \mathrm{~mm}$, has been confirmed as an independent predictor of both pathologic and clinical outcomes. $[4,22-25]$ With an increase in tumor size (>5 mm) on preoperative ultrasound, the risk for LNM in MPTC increases 2.9-3.7-fold. [4, 23] Such patients also have poor prognostic factors compared with those with a tumor diameter $<5 \mathrm{~mm},[24,25]$ further confirming that tumor size (> $5 \mathrm{~mm}$ ) is significantly correlated with CLNM. However, there remains no consensus on 
the prognostic significance of primary tumor size. [6, 26-29] In a study of 132 patients with low-risk MPTC, there was no relationship between tumor size and CLNM, although more tumors $\geq 7 \mathrm{~mm}$ tended to become enlarged than smaller tumors over the 4-year observation period. [26] In a more recent study, multivariate analysis found that a tumor diameter $\geq 9 \mathrm{~mm}$ at diagnosis was an independent risk factor for progression to clinical disease; however, this factor was not a predictor of tumor enlargement or CLNM alone. [6] In our study, we found that a largest diameter $\geq 10 \mathrm{~mm}$ was associated with CLNM, while the combination of tumor diameter and an $\mathrm{A} / \mathrm{T}$ ratio $\leq 0.828$ yielded a higher diagnostic performance, which is ascribed to tumor growth being more accurately modeled in measurements taken from multiple dimensions. [28] This result suggests that in these patients, careful preoperative examination of the central and lateral compartments and an aggressive surgical approach are needed.

TG is widely used for monitoring differentiated thyroid cancer recurrence and diagnosing LNM. [30, 31] However, whether preoperative serum TG could predict CLNM in PTC is still controversial. Kim et al. [32] discovered that a TG concentration $>63.4 \mathrm{ng} / \mathrm{mL}$ was useful in predicting $\operatorname{CLNM}(P<0.05,0 \mathrm{R}=9.412)$, while Patell et al. [33] showed that there was no correlation between TG concentration and CLNM. Although the mechanisms of how elevated TG affects CLNM remain unclear, our results indicated that the metastatic burden of the central tumor would increase the TG level. However, further research evaluating TG in aggressive disease is needed to enable the early detection of CLNM.

There are some limitations in this study that should be mentioned. First, the model was developed for classifying CLNM. Therefore, the diagnostic performance of the model in classifying lateral LNM could not be evaluated. However, in practice, information on lateral LNM is critical for surgical decision-making. Second, this model was not developed to localize cervical lymph nodes; therefore, users need to identify the affected lymph nodes on US. Third, this study was based on a single center, and an external validation study is needed to validate the predictive performance and generalizability of the model. Prospective and multi-institutional datasets are also needed in future studies.

\section{Conclusions}

In conclusion, this predictive model based on a combination of clinical and sonographic features has good performance for predicting CLNM in PTMC patients. Combining an A/T ratio $\leq 0.828$ and a largest anteroposterior diameter $\geq 10 \mathrm{~mm}$ play important role in determining CLNM. These findings could improve the management of MPTC by supporting clinicians and reducing the number of invasive surgical procedures performed for patients with low-risk thyroid cancers as well as help in clinical decision making.

\section{Abbreviations}

MPTC

micropapillary thyroid cancer

CLNM 
central lymph node metastasis

US

ultrasonography

ATA

American Thyroid Association

NCCN

National Comprehensive Cancer Network

$\mathrm{ML}$

Machine learning

AUC

area under the curve

CLND

central neck dissection

LND

lateral neck dissection

ETE

extrathyroidal extension

CT

calcitonin

PTH

parathyroid hormone

TG

thyroglobulin

TSH

thyroid-stimulating hormone

T3

triiodothyronine

FT3

free triiodothyronine

T4

thyroxine

FT4

free thyroxine

$\mathrm{A} / \mathrm{T}$

anteroposterior/transverse diameter

ROC

receiver operating characteristic

PPV

positive predictive value

NPV 
negative predictive value

AJCC

American Joint Committee on Cancer

\section{Declarations}

\section{Ethics approval and consent to participate}

This study was approved by the Institutional Ethics Committee of Jinling Hospital. This study kept confidentiality of patient data and strictly complied with the Declaration of Helsinki and its later amendments or comparable ethical standards.

\section{Consent for publication}

Written informed consent for publication of their clinical details and clinical images was obtained from all patients.

\section{Availability of data and materials}

The datasets used and/or analysed during the current study are available from the corresponding author on reasonable request.

\section{Competing interests}

The authors declare that they have no competing interests.

\section{Funding}

Not applicable.

\section{Authors' contributions}

All authors made substantive intellectual contributions to this study to qualify as authors. XCL and ZQR conceived of the design of the study. YB and ZZ modified the design of the study. SB and ZC performed the study, collected the data, and contributed to the design of the study. WXM analyzed the data. FXD drafted "Results," “Discussion," and "Conclusions" sections. LSY and SJY drafted the "Methods" sections. $\mathrm{XCL}$ and ZQR edited the manuscript. All authors read and approved the final manuscript. All authors have agreed to be accountable for all aspects of the work in ensuring that questions related to the accuracy or integrity of any part of the work are appropriately investigated and resolved.

\section{Acknowledgements}

Not applicable. 


\section{References}

1. Wang C, Crapo LM. The epidemiology of thyroid disease and implications for screening. Endocrinol Metab Clin North Am. 1997; 26(1):189-218.

2. Harach HR, Franssila KO, Wasenius VM. Occult papillary carcinoma of the thyroid. A "normal" finding in Finland. A systematic autopsy study. Cancer. 1985; 56(3):531-8.

3. Tao Y, Wang C, Li L, Xing H, Bai Y, Han B, Liu Z, Yang X, Zhu S. Clinicopathological features for predicting central and lateral lymph node metastasis in papillary thyroid microcarcinoma: Analysis of 66 cases that underwent central and lateral lymph node dissection. Mol Clin Oncol. 2017; 6(1):4955.

4. Lim YC, Choi EC, Yoon YH, Kim EH, Koo BS. Central lymph node metastases in unilateral papillary thyroid microcarcinoma. Br J Surg. 2009; 96(3):253-257.

5. Wada N, Duh QY, Sugino K, Iwasaki H, Kameyama K, Mimura T, Ito K, Takami H, Takanashi Y. Lymph node metastasis from 259 papillary thyroid microcarcinomas: frequency, pattern of occurrence and recurrence, and optimal strategy for neck dissection. Ann Surg. 2003; 237(3):399-407.

6. Ito Y, Miyauchi A, Kihara M, Higashiyama T, Kobayashi K, Miya A. Patient age is significantly related to the progression of papillary microcarcinoma of the thyroid under observation. Thyroid. 2014; 24(1):27-34.

7. Cho SY, Lee TH, Ku YH, Kim HI, Lee GH, Kim MJ. Central lymph node metastasis in papillary thyroid microcarcinoma can be stratified according to the number, the size of metastatic foci, and the presence of desmoplasia. Surgery. 2015; 157(1):111-8.

8. Mazzaferri EL, Doherty GM, Steward DL. The pros and cons of prophylactic central compartment lymph node dissection for papillary thyroid carcinoma. Thyroid. 2009; 19(7):683-9.

9. Hwang HS, Orloff LA. Efficacy of preoperative neck ultrasound in the detection of cervical lymph node metastasis from thyroid cancer. Laryngoscope. 2011; 121(3):487-91.

10. Haugen BR, Alexander EK, Bible KC, Doherty GM, Mandel SJ, Nikiforov YE, Pacini F, Randolph GW, Sawka AM, Schlumberger M, et al. 2015 American Thyroid Association Management Guidelines for Adult Patients with Thyroid Nodules and Differentiated Thyroid Cancer: The American Thyroid Association Guidelines Task Force on Thyroid Nodules and Differentiated Thyroid Cancer. Thyroid. 2016; 26(1):1-133.

11. Yeh MW, Bauer AJ, Bernet VA, Ferris RL, Loevner LA, Mandel SJ, Orloff LA, Randolph GW, Steward DL, American Thyroid Association Surgical Affairs Committee Writing Task F. American Thyroid Association statement on preoperative imaging for thyroid cancer surgery. Thyroid. 2015; 25(1):314.

12. Solorzano CC, Carneiro DM, Ramirez M, Lee TM, Irvin GL, 3rd. Surgeon-performed ultrasound in the management of thyroid malignancy. Am Surg. 2004; 70(7):576-80; discussion 580-72.

13. Stulak JM, Grant CS, Farley DR, Thompson GB, van Heerden JA, Hay ID, Reading CC, Charboneau JW. Value of preoperative ultrasonography in the surgical management of initial and reoperative 
papillary thyroid cancer. Arch Surg. 2006; 141(5):489-494; discussion 494-86.

14. O'Connell K, Yen TW, Quiroz F, Evans DB, Wang TS. The utility of routine preoperative cervical ultrasonography in patients undergoing thyroidectomy for differentiated thyroid cancer. Surgery. 2013; 154(4):697-701; discussion 701-693.

15. Liu T, Ge X, Yu J, Guo Y, Wang Y, Wang W, Cui L. Comparison of the application of B-mode and strain elastography ultrasound in the estimation of lymph node metastasis of papillary thyroid carcinoma based on a radiomics approach. Int J Comput Assist Radiol Surg. 2018; 13(10):1617-27.

16. Liu T, Zhou S, Yu J, Guo Y, Wang Y, Zhou J, Chang C. Prediction of Lymph Node Metastasis in Patients With Papillary Thyroid Carcinoma: A Radiomics Method Based on Preoperative Ultrasound Images. Technol Cancer Res Treat. 2019; 18:1533033819831713.

17. Zou Y, Shi Y, Liu J, Cui G, Yang Z, Liu M, Sun F. A Comparative Analysis of Six Machine Learning Models Based on Ultrasound to Distinguish the Possibility of Central Cervical Lymph Node Metastasis in Patients With Papillary Thyroid Carcinoma. Front Oncol. 2021; 11:656127.

18. American Institute of Ultrasound in M. AlUM Practice Guideline for the performance of thyroid and parathyroid ultrasound examination. J Ultrasound Med. 2003; 22(10):1126-30.

19. Koshkina A, Fazelzad R, Sugitani I, Miyauchi A, Thabane L, Goldstein DP, Ghai S, Sawka AM. Association of Patient Age With Progression of Low-risk Papillary Thyroid Carcinoma Under Active Surveillance: A Systematic Review and Meta-analysis. JAMA Otolaryngol Head Neck Surg. 2020; 146(6):552-60.

20. Miyauchi A, Kudo T, Ito Y, Oda H, Sasai H, Higashiyama T, Fukushima M, Masuoka H, Kihara M, Miya A. Estimation of the lifetime probability of disease progression of papillary microcarcinoma of the thyroid during active surveillance. Surgery. 2018; 163(1):48-52.

21. Nam SH, Bae MR, Roh JL, Gong G, Cho KJ, Choi SH, Nam SY, Kim SY. A comparison of the 7th and 8th editions of the AJCC staging system in terms of predicting recurrence and survival in patients with papillary thyroid carcinoma. Oral Oncol. 2018; 87:158-64.

22. Medas F, Canu GL, Cappellacci F, Boi F, Lai ML, Erdas E, Calo PG. Predictive Factors of Lymph Node Metastasis in Patients With Papillary Microcarcinoma of the Thyroid: Retrospective Analysis on 293 Cases. Front Endocrinol (Lausanne). 2020; 11:551.

23. Yang Y, Chen C, Chen Z, Jiang J, Chen Y, Jin L, Guo G, Zhang X, Ye T. Prediction of central compartment lymph node metastasis in papillary thyroid microcarcinoma. Clin Endocrinol (Oxf). 2014; 81(2):282-8.

24. Machens $A$, Holzhausen $H J$, Dralle $H$. The prognostic value of primary tumor size in papillary and follicular thyroid carcinoma. Cancer. 2005; 103(11):2269-73.

25. Roti E, Rossi R, Trasforini G, Bertelli F, Ambrosio MR, Busutti L, Pearce EN, Braverman LE, Degli Uberti EC. Clinical and histological characteristics of papillary thyroid microcarcinoma: results of a retrospective study in 243 patients. J Clin Endocrinol Metab. 2006; 91(6):2171-8.

26. Ito Y, Uruno T, Nakano K, Takamura Y, Miya A, Kobayashi K, Yokozawa T, Matsuzuka F, Kuma S, Kuma K, Miyauchi A. An observation trial without surgical treatment in patients with papillary 
microcarcinoma of the thyroid. Thyroid. 2003; 13(4):381-7.

27. Ito Y, Tomoda C, Uruno T, Takamura Y, Miya A, Kobayashi K, Matsuzuka F, Kuma K, Miyauchi A. Preoperative ultrasonographic examination for lymph node metastasis: usefulness when designing lymph node dissection for papillary microcarcinoma of the thyroid. World J Surg. 2004; 28(5):498501.

28. Tuttle RM, Fagin JA, Minkowitz G, Wong RJ, Roman B, Patel S, Untch B, Ganly I, Shaha AR, Shah JP, et al. Natural History and Tumor Volume Kinetics of Papillary Thyroid Cancers During Active Surveillance. JAMA Otolaryngol Head Neck Surg. 2017; 143(10):1015-20.

29. Kwon H, Oh HS, Kim M, Park S, Jeon MJ, Kim WG, Kim WB, Shong YK, Song DE, Baek JH, et al. Active Surveillance for Patients With Papillary Thyroid Microcarcinoma: A Single Center's Experience in Korea. J Clin Endocrinol Metab. 2017; 102(6):1917-25.

30. Matana A, Popovic M, Boutin T, Torlak V, Brdar D, Gunjaca I, Kolcic I, Boraska Perica V, Punda A, Rudan I, et al. Genetic Variants in the ST6GAL1 Gene Are Associated with Thyroglobulin Plasma Level in Healthy Individuals. Thyroid. 2019; 29(6):886-93.

31. Lee SH, Roh JL, Gong G, Cho KJ, Choi SH, Nam SY, Kim SY. Risk Factors for Recurrence After Treatment of N1b Papillary Thyroid Carcinoma. Ann Surg. 2019; 269(5):966-71.

32. Kim H, Kim YN, Kim HI, Park SY, Choe JH, Kim JH, Kim JS, Chung JH, Kim TH, Kim SW. Preoperative serum thyroglobulin predicts initial distant metastasis in patients with differentiated thyroid cancer. Sci Rep. 2017; 7(1):16955.

33. Patell R, Mikhael A, Tabet M, Bena J, Berber E, Nasr C. Assessing the utility of preoperative serum thyroglobulin in differentiated thyroid cancer: a retrospective cohort study. Endocrine. 2018; 61(3):506-10.

\section{Figures}



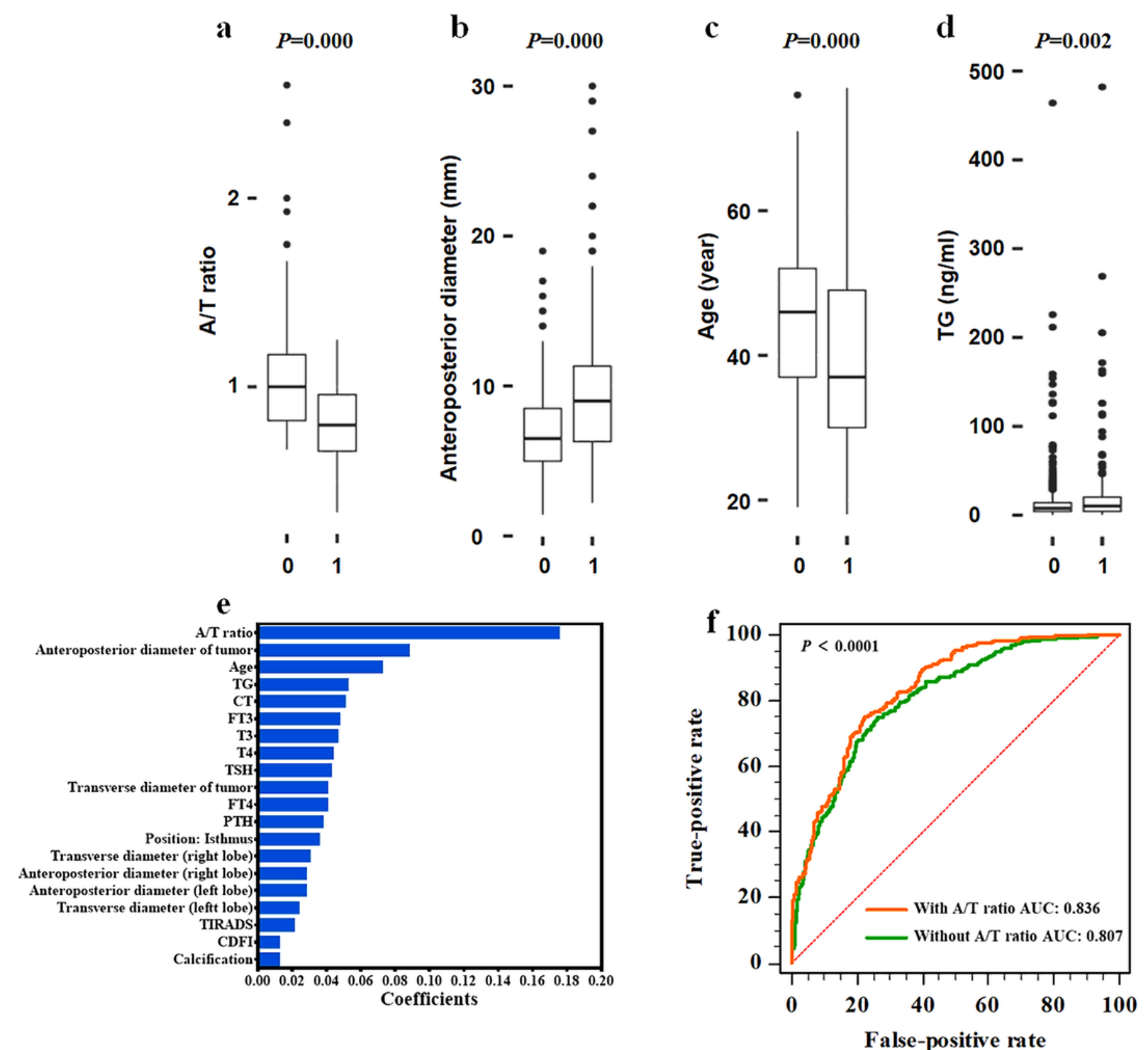

Figure 1

Box plots of the top weighted features and predictive performance of the random forest model for CLNM in MPTC patients. Patients with CLNM had a lower A/T ratio (a), higher anteroposterior diameter (b), younger age (c), and higher TG concentration (d) than those without CLNM. (e) The weighted features of the model. (f) The AUC of the model with A/T ratio and without A/T ratio for predicting CLNM were 0.836 , 0.807 , respectively. 

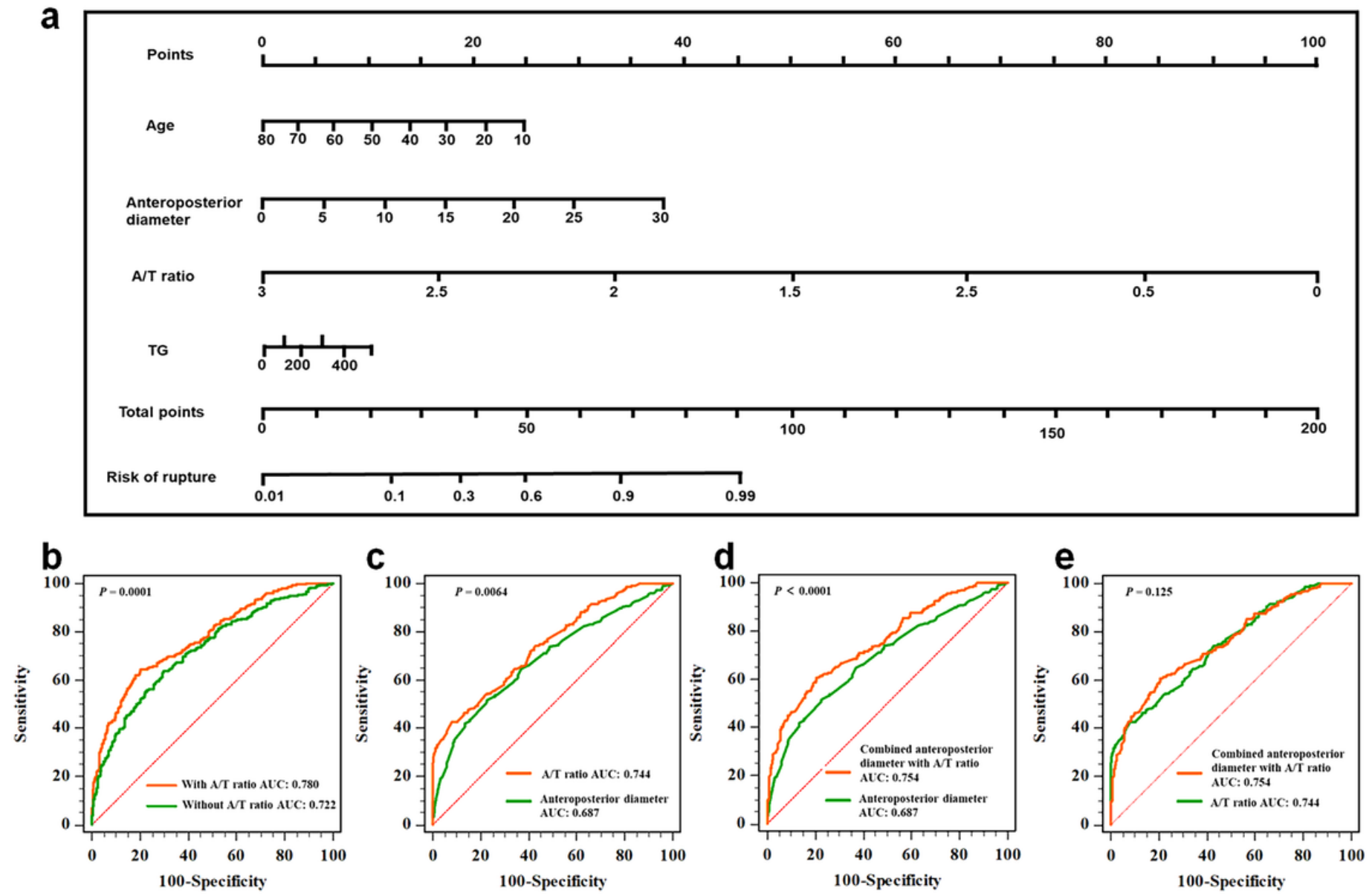

\section{Figure 2}

The nomogram and its diagnostic performance. (a) The nomogram was developed and included four factors (age, A/T ratio, anteroposterior diameter, and TG). (b) The ROC curve of the nomogram with and without A/T ratio for predicting CLNM. (c) The ROC curve of A/T ration alone and anteroposterior diameter alone as a single feature in the nomogram for predicting CLNM. (d) The ROC curve of the combination of $\mathrm{A} / \mathrm{T}$ and anteroposterior diameter, and anteroposterior diameter as a single feature in the nomogram for predicting CLNM. (e) The ROC curve of the anteroposterior diameter combined with the $\mathrm{A} / \mathrm{T}$ ratio, and $\mathrm{A} / \mathrm{T}$ ratio as a single feature in the nomogram for predicting CLNM. 

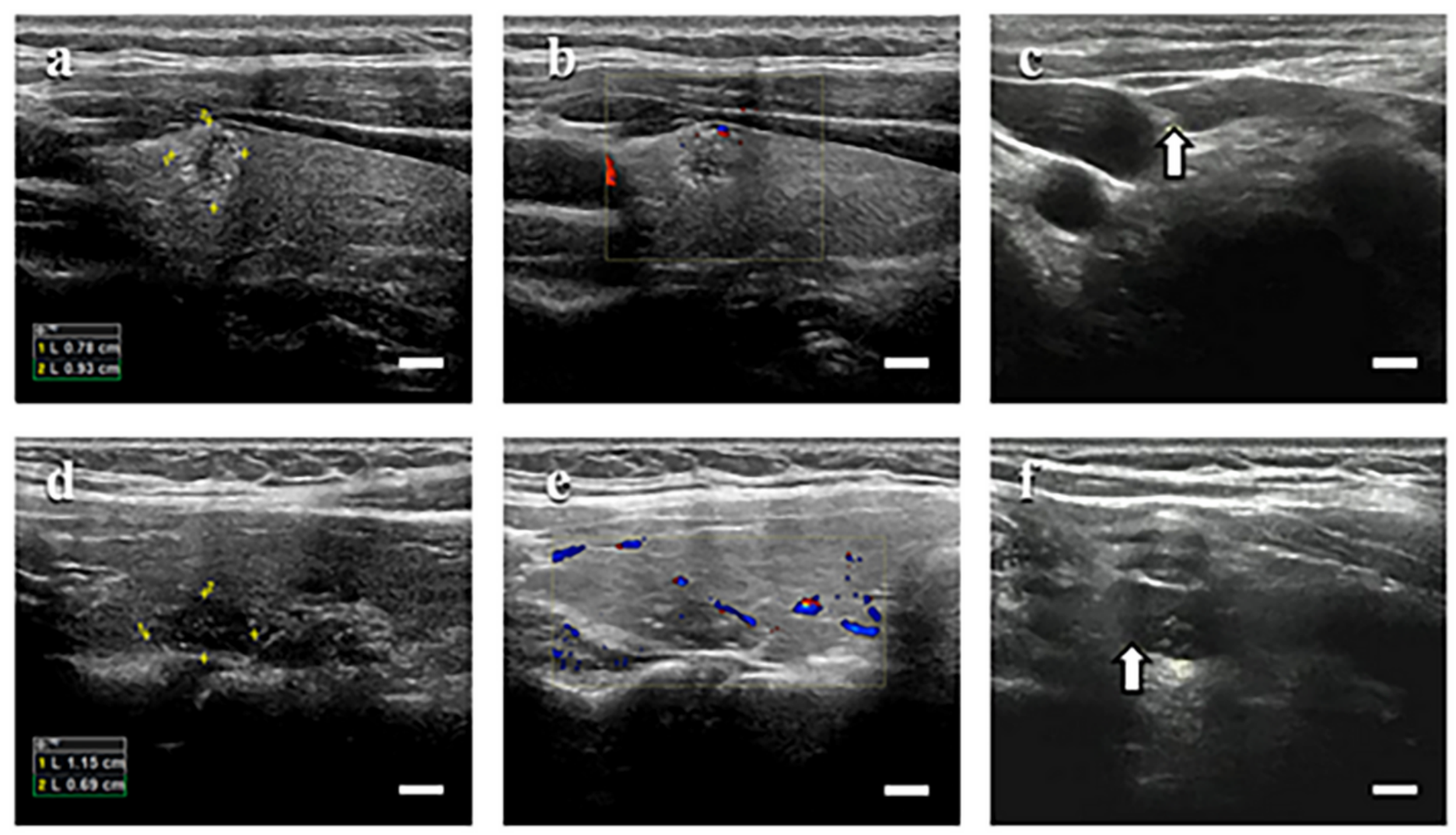

\section{Figure 3}

Definitions of the tumor size measurements on US images for predicting CLNM. (a) MPTC of the left lobe in a 43-year-old woman. The lesion was $7.8 \times 9.3 \mathrm{~mm}$ in size, had an A/T ratio $=1.19$, had an irregular shape, had an unclear margin, and had multiple calcifications that were observed on US images. (b) CDFI showed and Adler grade of 2. (c) The US image indicated a suspicion of CLNM, while the pathological examination confirmed that CLNM was not present. (d) MPTC of the right lobe in a 35-year-old woman. The lesion was $11.5 \times 6.9 \mathrm{~mm}$ in size, had an $\mathrm{A} / \mathrm{T}$ ratio $=0.60$, had an irregular shape, had unclear margins, and no calcification were observed on US. Scale bar: $5 \mathrm{~mm}$. 